\title{
Determination of the mechanical properties of amorphous materials through instrumented nanoindentation
}

\author{
M. Rodríguez ${ }^{\text {a }}$ J.M. Molina-Aldareguía ${ }^{\text {a }}$, C. González ${ }^{\text {a,b }}$, J. LLorca ${ }^{\text {a,b,* }}$
}

\begin{abstract}
A novel methodology based on instrumented indentation is developed to determine the mechanical properties of amorphous materials which present cohesive-frictional behaviour. The approach is based on the concept of a universal hardness equation, which results from the assumption of a characteristic indentation pressure proportional to the hardness. The actual universal hardness equation is obtained from a detailed finite element analysis of the process of sharp indentation for a very wide range of material properties, and the inverse problem (i.e. how to extract the elastic modulus, the compressive yield strength and the friction angle) from instrumented indentation is solved. The applicability and limitations of the novel approach are highlighted. Finally, the model is validated against experimental data in metallic and ceramic glasses as well as polymers, covering a wide range of amorphous materials in terms of elastic modulus, yield strength and friction angle.
\end{abstract}

\section{Introduction}

Instrumented indentation, in which the load and depth of indentation are continuously recorded during the indentation process, is a simple testing technique, and a large research effort has been devoted to obtaining the elasto-plastic properties of materials from this test. Most of these studies focused on polycrystalline solids, in which the flow stress follows the von Mises criterion and is equivalent under uniaxial tension and compression [1-3]. This criterion is not representative, however, of the behaviour of amorphous solids such as ceramic glasses, bulk metallic glasses and both thermoset and thermoplastic polymers. They usually display brittle behaviour in tension, while deforming plastically in compression and/or shear, and the flow stress depends on the hydrostatic pressure [4]. As a result, the elasto-plastic properties of these cohesivefrictional materials are difficult to measure by conventional methods such as tensile testing, owing to their inherent brittleness. In contrast, they are readily deformed by indentation, owing to the large hydrostatic compression under the indenter tip, the small volume of deformed material and the constraint of the surrounding material. Therefore, instrumented indentation stands as an ideal technique for characterizing the elasto-plastic constitutive behaviour of cohesive-frictional materials.

Extracting material properties from instrumented indentation is, however, extremely challenging, because there is no one-to-one correspondence between the indentation curve and the material properties [3]. One of the main difficulties in relating both is found in the uncertainty in how the material accommodates the volume displaced by the 
indenter. On the one hand, purely elastic material accommodates the displaced volume by the elastic deformation of the surrounding material, leading to a sink-in of the material around the indenter and reducing the actual contact area. On the other hand, plastic flow displaces the material upwards around the tip, and the resulting pileup around the indenter will increase the actual contact area in very ductile materials. Engineering materials normally present intermediate behaviour between these extremes, which has been extensively studied in elasto-plastic solids, following the von Mises yield criterion [5]. However, very little is known about this phenomenon for cohesive-frictional materials. Giannakopoulos and Larsson [6] and Vaidyanathan et al. [7] studied the pyramidal indentation of cohesive-frictional materials using the finite element method and, more recently, Narasimhan [8] and Patnaik et al. [9] analysed the applicability of the expanding cavity model $[10,11]$ to studying the conical and spherical indentation response of elasto-plastic materials in which the flow stress depends on the hydrostatic pressure. However, none of these studies focused on the determination of the constitutive response of the material from the indentation curve, and there is no reliable methodology for estimating the yield stress and pressure sensitivity in amorphous materials from instrumented indentation. In fact, yield stresses of polymers and metallic glasses derived from indentation studies led to unreasonably large values [12].

This work aims to cover this gap by establishing a methodology to determine the constitutive elasto-plastic behaviour of cohesive-frictional materials from instrumented indentation. The methodology is based on a universal hardness equation for cohesive-frictional materials based on the novel concept of the characteristic indentation pressure. The actual universal hardness equation (independent of the ratio of flow stress to elastic modulus and of the pressure sensitivity) was obtained from a systematic numerical simulation of the process of sharp indentation in cohesive-frictional materials. Based on this universal hardness equation, the inverse problem was solved to derive the material properties (elastic modulus, flow stress and pressure sensitivity) from the indentation curve, and the validity and limitations of the new methodology were established. Finally, the method was validated experimentally in various amorphous materials that follow a cohesive-frictional behaviour (metallic and ceramic glasses as well as polymers) and with very different flow stress/elastic modulus ratios.

\section{Theoretical considerations}

\subsection{Constitutive behaviour of cohesive-frictional materials}

Amorphous materials deform plastically in compression by the formation of shear bands. Further loading leads to the localization of the deformation within the shear band (or in an array of parallel bands) without any strain hardening. There is a wide body of experimental evidence showing that the yield criterion for shear band formation in metallic $[13-16]$ and ceramic glasses $[17,18]$ as well as in polymers [19-22] depends on the hydrostatic stress and that the yield surface of these materials is adequately represented by either the Mohr-Coulomb or the Drucker-Prager yield criterion. Mathematically, the yield surface $(\Phi=0)$ is given by [23]

$\Phi=\sqrt{3 J_{2}}-d+\frac{I_{1}}{3} \tan \beta=0$

where $I_{1}$ stands for the first invariant of the Cauchy stress tensor $\sigma_{i j}$, and $J_{2}$ for the second invariant of the deviatoric part of the Cauchy stress tensor according to

$I_{1}=\sigma_{i i} \quad \sigma_{i j}^{\prime}=\sigma_{i j}-\frac{I_{1}}{3} \quad J_{2}=\frac{1}{2} \sigma_{i j}^{\prime} \sigma_{j i}^{\prime}$

The elasto-plastic behaviour of the material is thus characterized by the two elastic constants $E$ and $v$ (assuming isotropic behaviour) and the two parameters which dictate the onset of plastic deformation, namely the cohesion $d$ and the friction angle $\beta$, which controls the pressure-sensitivity of the material. Eq. (1) represents a conical surface in principal stress space with the vertex on the hydrostatic stress axis. The trace of the yield surface on the deviatoric plane is circular and leads to the von Mises yield criterion (with yield stress equal to $d$ ) in the particular case of $\beta=0^{\circ}$.

\subsection{Analysis of instrumented indentation test}

The output of an instrumented indentation test is the load displacement curve during loading and unloading of the indenter, as shown in Fig. 1. The main parameters obtained from the test are the maximum indentation load $P_{\max }$, the maximum indentation depth $h_{\max }$, and the elastic stiffness upon unloading $S$. Another interesting factor is the elastic energy/total energy ratio, $W_{e} / W_{t}=W_{e} /$ $\left(W_{e}+W_{p}\right)$, where the elastic energy $W_{e}$ and the energy dissipated by plastic deformation $W_{p}$ are shown in Fig. 1. It

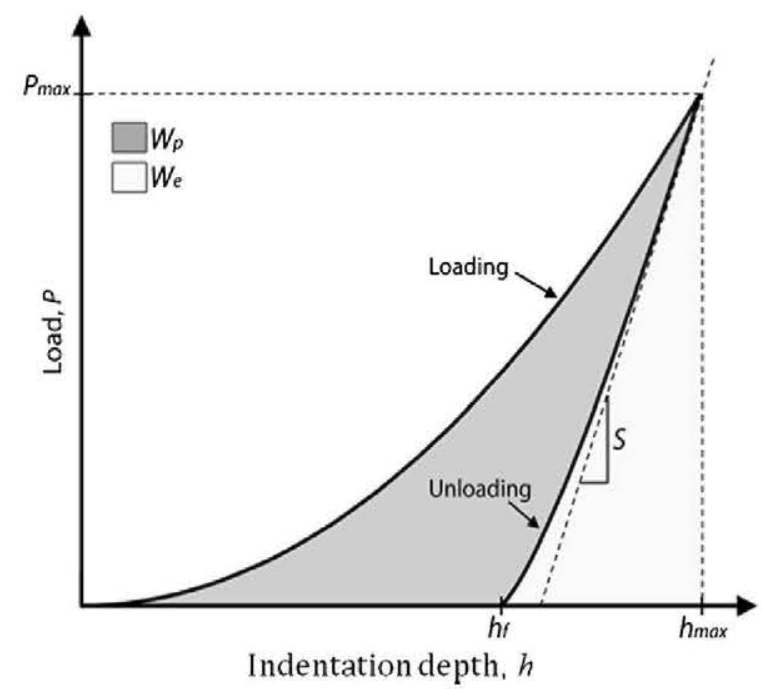

Fig. 1. Schematic of a load-indentation depth curve showing the most relevant experimental parameters that can be obtained from the test. 
was demonstrated [3] that only two of these parameters are independent, and thus extra hypotheses are necessary to determine the material properties from a single pyramidal indentation test, as the constitutive behaviour of an elastoplastic solid following the Drucker-Prager model is described by four independent constants (see Section 2.1). This additional information is provided by the characteristic indentation pressure concept, which is presented in Section 2.3 .

Another challenge of the instrumented indentation test is the estimation of the actual contact area. This is normally accomplished by means of analytical methods, of which the most widely used is due to Oliver and Pharr [24]. This method provides a good approximation of the actual contact area when the material surrounding the indenter sinks in, but it does not consider situations that lead to pile-up of the material around the indenter, as schematically represented in Fig. 2 [5]. The development of material pile-up or sink-in depends on the indenter geometry and the material properties, and the contact area will be severely underestimated if pile-up is dominant (as is the case in metallic glasses and other amorphous materials [25]).

To avoid the uncertainty arising from the determination of the contact area, the present methodology uses the apparent hardness $H_{a p}$ instead of the actual hardness $H$ as the basis of the analysis. They are given by

$H_{a p}=\frac{P_{\max }}{A_{a p}} \quad H=\frac{P_{\max }}{A}$

where $A_{a p}$ and $A$ stand for the apparent and actual contact area, respectively. The apparent contact area is directly obtained from the shape of the indenter tip as a function of the maximum depth $A_{a p}=f\left(h_{\max }\right)$, and it is related to the actual contact area through the pile-up parameter $c_{p}$, defined as

$c_{p}=\sqrt{\frac{A}{A_{a p}}}$

Hence, $c_{p}>1$ indicates that material piles up around the indenter, while $c_{p}<1$ is representative of a sink-in phenomenon, as illustrated in Fig. 2.

Once $c_{p}$ is known, it is possible to determine the elastic properties of the material from the reduced elastic modulus $E^{*}$, which is given by

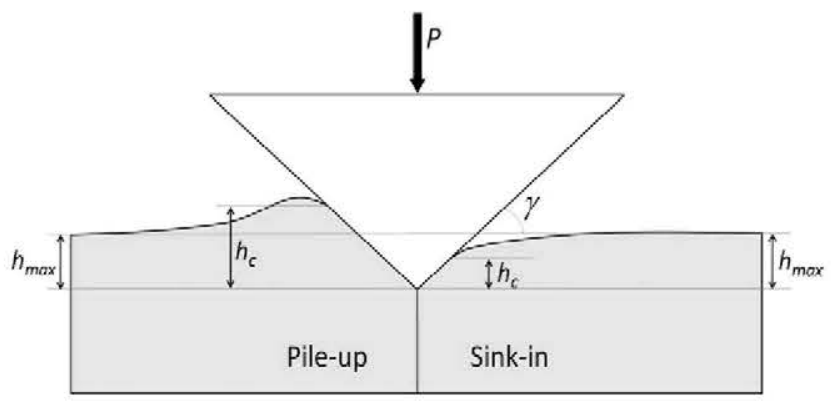

Fig. 2. Schematic diagram showing pile-up/sink-in effects around the indenter tip.
$\frac{1}{E^{*}}=\frac{1-v^{2}}{E}+\frac{1-v_{i}^{2}}{E_{i}}$

and accounts for the elastic deformation of the indenter through its elastic constants $\left(E_{i}, v_{i}\right) . E^{*}$ can be obtained from the unloading stiffness $S$ according to Sneddon's equation [37]:

$E^{*}=\frac{S \sqrt{\pi}}{2 \sqrt{A}}=\frac{S \sqrt{\pi}}{2 c_{p} \sqrt{A_{a p}}}$

The determination of the cohesion $d$ and of the frictional angle $\beta$ from the actual hardness $H$ is not, however, immediate, as shown below.

\subsection{The concept of characteristic indentation pressure: a novel approach}

The average hardness measured in an indentation test is always higher than the uniaxial compressive yield stress $\sigma_{y c}$ because of the confining pressure generated by the elastically strained material around the indenter. The ratio between the indentation mean contact pressure and the uniaxial compressive yield stress is called the constraint factor $C$. For very ductile metals (with a plasticity index $\sigma_{y c}$ l $\left.E^{*}<0.001\right), \quad C \approx 2.5-3$ is generally considered to be appropriate, in agreement with Lockett's solution [26] for rigid-perfectly plastic materials. This value is only valid, however, when the material is in the fully plastic regime.

For elasto-plastic materials following the von Mises yield criterion, Johnson [11] concluded that the constraint factor depends on the plasticity index $\sigma_{y c} / E^{*}$. If $\sigma_{y c} / E^{*}<0.01$, the indentation is in the fully plastic regime and therefore $C \approx 2.5-3$. The elastic deformation is not negligible, however, for higher values of $\sigma_{y c} / E^{*}$, and the constraint factor decreases. Similarly, strain hardening also affects the constraint factor, but it shows an upper limit of 3 for elastoplastic materials following the von Mises yield criterion [2].

Few analyses of the constraint factor are available for cohesive-frictional materials, in which the flow stress depends on the hydrostatic stress. Keryvin et al. [15] studied the case of spherical indentations, while Ganneau et al. [27] dealt with pyramidal indentations, but their investigation was limited to rigid-plastic materials (very small $\sigma_{y c} d E^{*}$ ). Thus, there is not a systematic study which relates the indentation hardness to the compressive yield strength and the friction angle, and that is the objective of this section. The equations governing the hardness in von Mises elasto-plastic materials are first reviewed and a novel method is proposed to extend the analysis to cohesive-frictional solids.

In the case of von Mises materials, it is well established from dimensional analysis [28] that the relationship between the hardness $H$ and the yield stress $\sigma_{y}$ for a given conical indenter angle $\gamma$ adopts the form

$\frac{H}{\sigma_{y c}}=f\left(\frac{\sigma_{y c}}{E^{*}}\right)$ 
where the constraint function $f$ depends on the plasticity index. Eq. (7) was generalized for strain-hardening materials by Mata et al. [29] by replacing the yield stress $\sigma_{y}$ with the stress level in the uniaxial stress-strain curve at a characteristic plastic strain $\varepsilon_{r}$, which is usually $\sim 0.01$ for Berkovich indenters. The concept of the characteristic indentation strain was first introduced by Tabor [30] for fully plastic indentations and extended by Johnson [10] and Mata et al. [29] for the case of elasto-plastic indentations.

In the case of cohesive-frictional materials, Eq. (7) is expected to take the following form:

$\frac{H}{\sigma_{y c}}=f\left(\frac{\sigma_{y c}}{E^{*}}, \beta\right)$

where $\sigma_{y c}$ stands for the uniaxial compressive yield stress, and $\beta$ the pressure sensitivity index. Inspired by the above, the concept of a characteristic indentation stress $\sigma_{r}$ is postulated for cohesive-frictional materials. That is, the existence of a characteristic hydrostatic stress, $\sigma_{h}$ is assumed, induced by the conical indenter, which is related to the characteristic indentation stress according to (Eq. (1))

$\sigma_{r}=d-\sigma_{h} \tan \beta$

Furthermore, and since the hardness is a measurement of the average pressure applied on the contact area [10], it is postulated that the characteristic hydrostatic stress should be proportional to the hardness $H$, and hence Eq. (9) can be rewritten as

$\sigma_{r}=d-a H \tan \beta$

where $a$ is a proportionality constant that will be obtained from finite element simulations of the indentation process. If the assumption is correct, Eq. (8) can be rewritten as

$\frac{H}{\sigma_{r}}=f\left(\frac{\sigma_{r}}{E^{*}}\right)$

where the effect of the friction angle is already incorporated into $\sigma_{r}$, and the constraint function $f$ should only depend on the plasticity index $\sigma_{r} / E^{*}$ and not on $\beta$. The validity of this assumption was demonstrated from the results of the numerical analyses of the indentation process, as shown below.

\section{Numerical analysis of indentation}

\subsection{Finite element model}

The indentation process was simulated by means of the finite element method, assuming axial symmetry around the indenter axis (Fig. 3). The conical indenter was rigid with a semi-apex angle $\theta=70.3^{\circ}$. This conical indenter has the same area-to-depth ratio as the actual Berkovich indenter, and it has been demonstrated that the indentation response of both indenters is equivalent for a wide range of material properties [31,32].

The material was discretized with four-node bilinear axisymmetric elements with full integration (CAX4). The model dimensions were large enough to neglect boundary

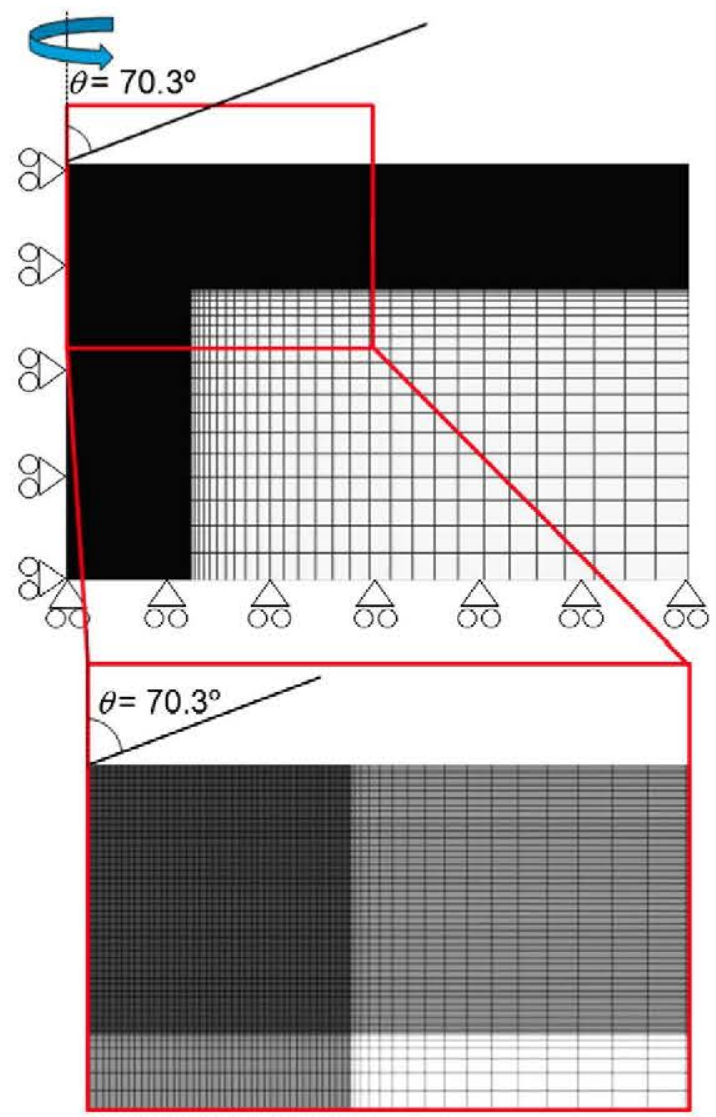

Fig. 3. Discretization and boundary conditions of the axisymmetric model to simulate a conical indentation, equivalent to a pyramidal Berkovich tip.

effects, and the mesh was refined around the indenter, as shown in Fig. 3 together with the boundary conditions. The minimum element size was selected in each case to maintain $\sim 40$ elements in contact with the indenter at the maximum indentation depth. It was checked that further mesh refinement only marginally affected the load-indentation curve. In all cases, the contact between the indenter and the material was assumed to be frictionless, because the effect of friction on the indentation response can be neglected for blunt tips $[10,33]$. To support this assumption, simulations with a coefficient of friction of 0.3 between the indenter and the material were carried out in the worst case scenario, i.e. a rigid perfectly plastic von Mises material. The effect of friction led to an increase in hardness below $10 \%$.

Most of the simulations were carried using Abaqus/ Standard within the framework of the finite deformations theory with the initial unstressed state as reference. However, element distortion was too large to provide accurate results of the pile-up when the plasticity index was very low. In these cases, simulations were carried out using Abaqus/Explicit with four-node axisymmetric bilinear elements with reduced integration and hourglass control (CAX4R) together with the arbitrary Lagrangian-Eulerian mesh adaptivity option. The curves obtained with the explicit and implicit algorithms led to the same results when comparison was possible. 
The indented material was modelled as an isotropic, elasto-plastic solid without hardening, following the DruckerPrager yield criterion. A non-associative flow rule was used to compute the directions of plastic flow in the stress space, and rate effects were neglected [34]. Simulations were carried out for a wide range of properties with $0.005 \leqslant \sigma_{y c} / E^{*} \leqslant 0.1$ and $0 \leqslant \beta \leqslant 48^{\circ}$. The results were assumed to depend on Young's modulus and Poisson's ratio only through $\sigma_{y d} / E^{*}$.

\subsection{Effect of the friction angle on the indentation response}

The friction angle had a very large effect on the indentation response, especially for small values of the plasticity index $\sigma_{y c} / E^{*}$. For instance, the maximum indentation load increased by a factor of 2 for soft materials in the fully plastic regime $\left(\sigma_{y c} / E^{*}=0.002\right)$ when the frictional angle increased from $0^{\circ}$ to $42^{\circ}$ (Fig. 4a). The effect of $\beta$ was noticeable, but smaller when $\sigma_{y c} / E^{*}=0.02$, which stands for the elasto-plastic regime (Fig. 4b), while it was negligible when the behaviour of the material under the indenter was elastic $\left(\sigma_{y c} / E^{*}=0.2\right.$, Fig. $\left.4 \mathrm{c}\right)$.

This behaviour can be easily understood, owing to the effect of the hydrostatic pressure on the flow stress. The higher the frictional angle $\beta$, the higher the von Mises equivalent stress $\left(\sqrt{3 J_{2}}\right)$ necessary to promote plastic deformation under the indenter and, thus, the higher the indentation load (Fig. 5). These observations are qualitatively similar to those advanced by Vaidyanathan et al. [7], Giannakopoulos and Larsson [6], and Patnaik et al. [9] for Berkovich, Vickers and conical indenters, respectively.

\subsection{Effect of friction angle on hardness}

The evolution of the constraint factor $H / \sigma_{y c}$ with the plasticity index $\sigma_{y d} / E^{*}$ is plotted in Fig. 6 for material with different friction angle. These results present the three indentation regimes postulated by Johnson [10]. If $\sigma_{y c} /$ $E^{*}>0.1$, the deformation under the indenter is elastic and, therefore, the hardness (understood as the mean pressure of indentation) is independent of the flow stress and of the friction angle. In contrast, the indentation is in the fully plastic regime if $\sigma_{y c} / E^{*}$ is very low and the constraint factor tends towards a constant value. Finally, the constraint factor decreases with $\sigma_{y c} / E^{*}$ in the intermediate, elasto-plastic regime.

The numerical simulations presented in Fig. 6 show that the friction angle strongly influences both the constraint factor and the transition between the different indentation regimes. For instance, the fully plastic regime in non-frictional materials $\left(\beta=0^{\circ}\right)$ was attained for $\sigma_{y c} / E^{*}<0.01$ and the corresponding constraint factor was $\sim 2.8 \sigma_{y c}$, in agreement with previous studies. This fully plastic regime was not attained, however, for $\sigma_{y c} d E^{*}=0.001$ when $\beta=48^{\circ}$ and the corresponding constraint factor was $>10 \sigma_{y c}$.

These results can be used to test the validity of the assumption about the existence of a characteristic hydrostatic stress in Section 2.3 and to calculate the value
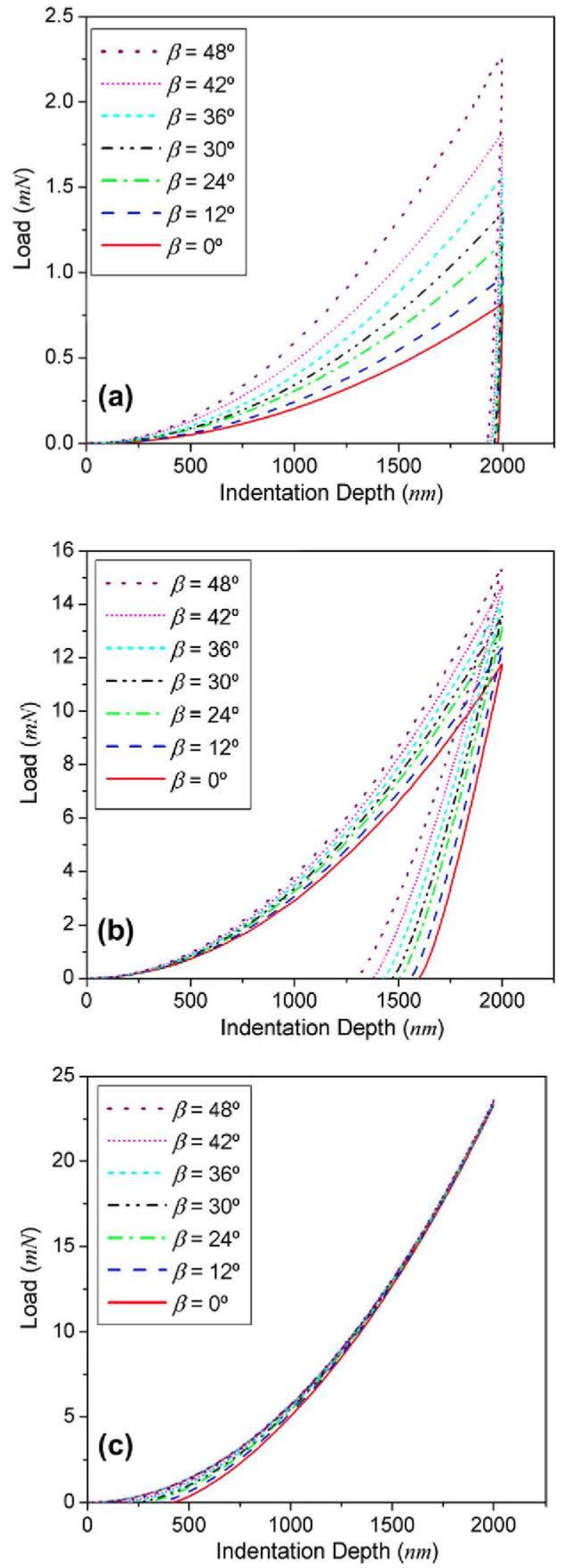

Fig. 4. Effect of the friction angle $\beta$ on the indentation load-depth curve for elasto-plastic materials following the Drucker-Prager yield criterion: (a) $\sigma_{y c} / E^{*}=0.002$, fully plastic regime; (b) $\sigma_{y c} / E^{*}=0.02$, elasto-plastic regime; (c) $\sigma_{y c} d E^{*}=0.2$, elastic regime. 


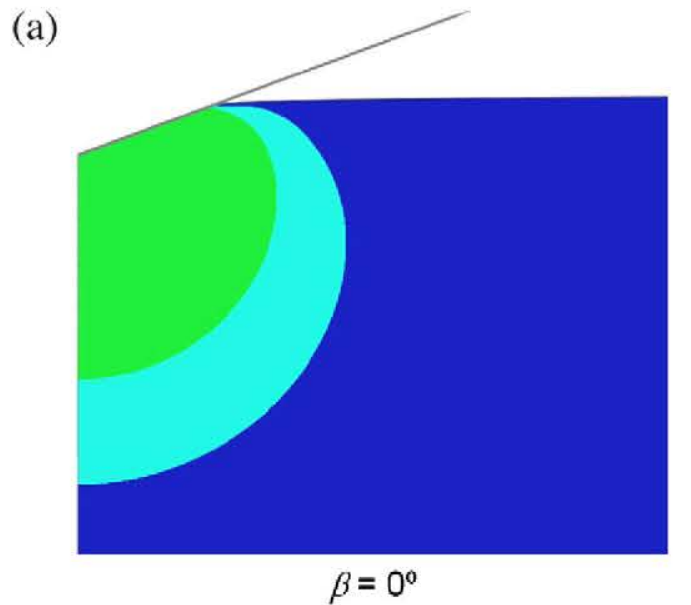

(b)

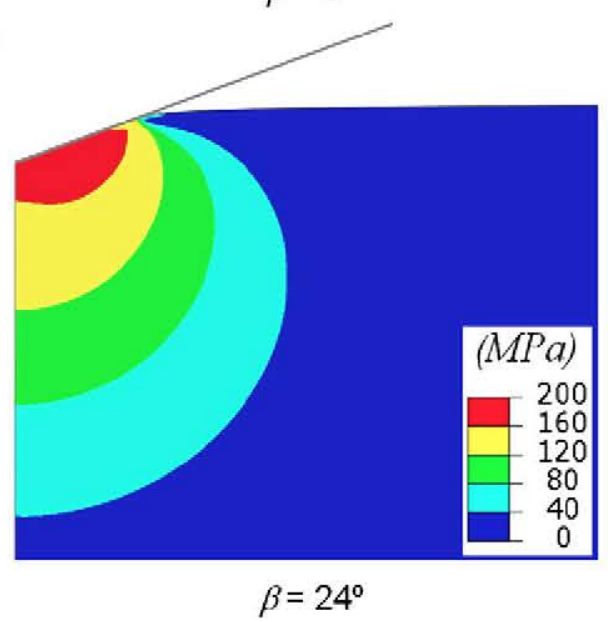

Fig. 5. Von Mises equivalent stress contours under the Berkovich indenter for a material with a plasticity index $\sigma_{y c} / E^{*}=0.04$ : (a) $\beta=0^{\circ}$; (b) $\beta=24^{\circ}$.

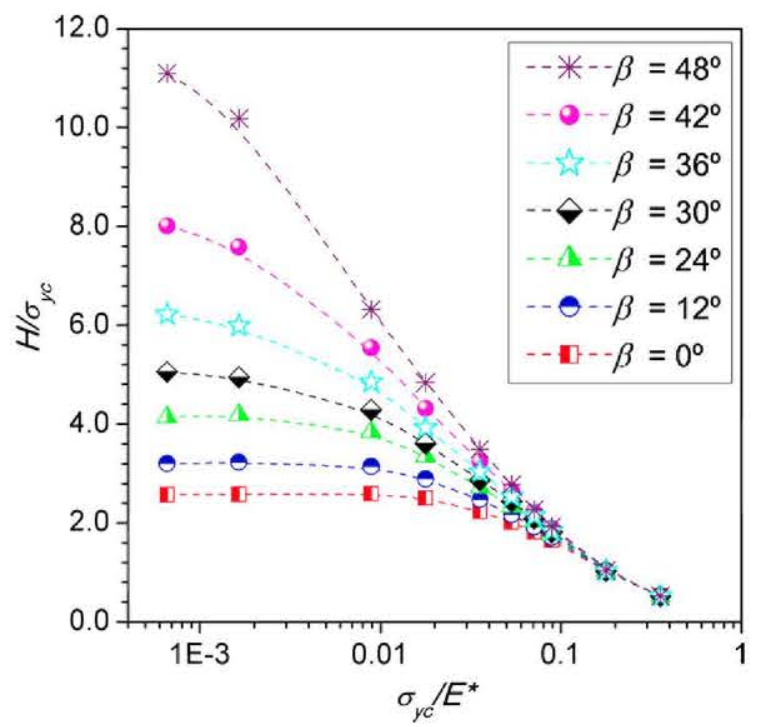

Fig. 6. Evolution of the constrain factor $H / \sigma_{y c}$ as a function of the plasticity index $\sigma_{y c} / E^{*}$ for materials with different Drucker-Prager friction angles.

of $a$ in Eq. (10). The latter can be inferred from the behaviour in the fully plastic regime, in which the constraint factor is constant. Thus, Eq. (11) can be written as

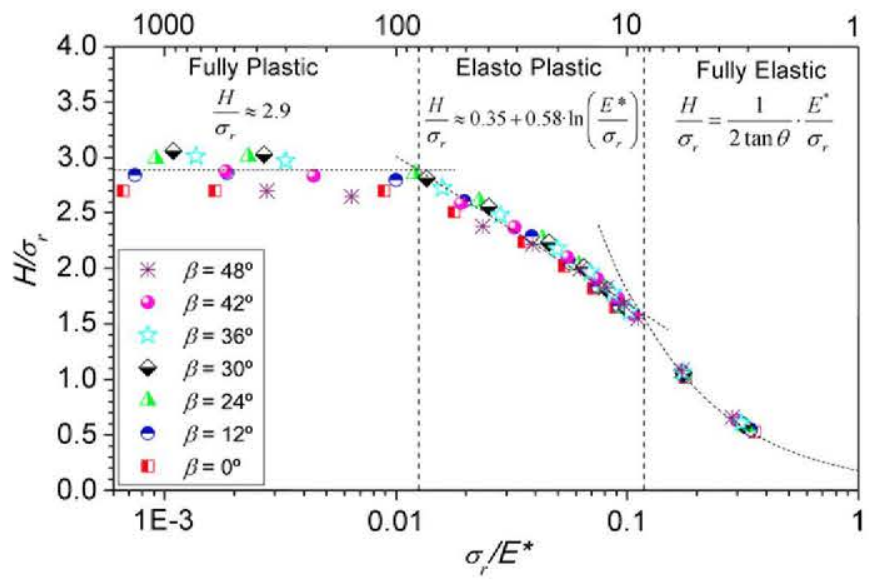

Fig. 7. Evolution of the constraint factor $H / \sigma_{r}$ as a function of the plasticity index $\sigma_{r} / E^{*}$ for materials with different Drucker-Prager friction angles.

$\frac{H}{\sigma_{r}}=C_{F P}$

where $C_{F P}$ represents the constraint factor in the fully plastic regime. From Eq. (10), this equation can be re-written as

$\frac{H}{d}=\frac{C_{F P}}{1+a C_{F P} \tan \beta}$

and $C_{F P} \approx 2.9$ and $a=0.29$ can be obtained by the least square fitting of this equation to the data corresponding to the fully plastic regime in Fig. $6 . C_{F P} \approx 2.9$ is in good agreement with the expected constraint factor for non-frictional materials. No reference exists for the characteristic hydrostatic stress in cohesive-frictional materials and a value of $\sigma_{h}=-0.29 H$ is obtained for Berkovich indenters.

Once $\sigma_{r}$ and $\beta$ are known, the uniaxial yield stress in compression $\sigma_{y c}$ can be easily calculated from Eqs. (1) and (9) as

$\sigma_{y c}=\frac{d}{1-\tan \beta / 2}=\frac{\sigma_{r}+a H \tan \beta}{1-\tan \beta / 2}$

The characteristic hydrostatic stress under the indenter has been estimated from the behaviour in the fully plastic regime, and it is necessary to assess the validity of this concept in other regimes. The results of the finite element analyses in Fig. 6 are re-plotted in Fig. 7 in terms of $\sigma_{r}$ instead $\sigma_{y c}$. All data, irrespective of friction angle $\beta$, lie on the same curve, and the limits between the fully plastic, the elastic-plastic and the elastic regimes are clearly recognized. Tabor's fully plastic regime develops for $\sigma_{r} /$ $E^{*}<0.012$, while the elastic regime is dominant for $\sigma_{r} /$ $E^{*}>0.1$. The elasto-plastic regime is found between these limits, and the decrease in the constraint factor with $\sigma_{r} / E^{*}$ is in good agreement with the results reported for $\beta=0^{\circ}$ $[2,29]$. In the elasto-plastic region, the variation in the constraint factor with the plasticity index can be fitted using the same expression derived by Hill [35], Marsh [36] and Johnson [10], leading to 


$$
\frac{H}{\sigma_{r}}=0.35+0.58 \ln \left(\frac{E^{*}}{\sigma_{r}}\right)
$$

Finally, the finite element results in the elastic regime follow the analytical expression for the elastic contact of a conical indenter on a semi-infinite half space [37]

$\frac{H}{\sigma_{r}}=\frac{\tan \gamma}{2} \frac{E^{*}}{\sigma_{r}}$

It should be noted, however, that it is extremely unlikely that real materials will lie within this region in the case of a blunt indenter (such as the one used here).

The current approach to developing a universal hardness equation for cohesive-frictional materials is debatable from a conceptual viewpoint, as the characteristic hydrostatic stress is derived from the data in the fully plastic regime. Nevertheless, the finite element simulations demonstrate that the substitution of $\sigma_{y c}$ by $\sigma_{r}$ in Eq. (8) certainly leads to very good correlation between the hardness and the mechanical properties of the material for a wide range of $E, \sigma_{y c}$ and $\beta$. This relationship is strictly valid for a conical indenter with $\gamma=19.7^{\circ}$, and hence applicable to pyramidal Vickers and Berkovich indentations in amorphous materials that display a non-hardening frictional behaviour.

\section{Determination of the mechanical properties from instrumented indentation}

The methodology presented above allows estimation of the hardness, provided that the material properties are known. The inverse problem is, however, more difficult for the reasons stated in Section 2.3, but it is far more interesting. The basic objective of this section is to determine the elastic $\left(E^{*}\right)$ and plastic $\left(\sigma_{y c}\right.$ and $\left.\beta\right)$ parameters which control the mechanical response of cohesive-frictional materials from the apparent hardness $H_{a p}$ (defined by $P_{\max }$ and $A_{\max }$ ), the elastic unloading stiffness $S$ and the $W_{e} / W_{t}$ ratio, which are readily obtained from instrumented indentation. The latter parameter is very useful, because $W_{e} / W_{t}$ is closely related to the plasticity index $\sigma_{r} / E^{*}$, which is not known a priori. $W_{e} / W_{t}=1$ in an elastic material, while $W_{e} / W_{t}$ will tend to 0 in the fully plastic regime, and Cheng and Cheng [28] proposed a linear relationship between both parameters. This may not be exact, but the data in Fig. 7 can be re-plotted in Fig. 8 as a function of $W_{e} / W_{t}$ instead of $\sigma_{r} /$ $E^{*}$, showing that the constraint factor $H / \sigma_{r}$ for the different friction angles lies within the same line up to $W_{e} / W_{t}<0.9$.

Taking this relationship into account, Fig. 9 re-plots the data in Fig. 6 as a function of $H_{a p}$ and $W_{e} / W_{t}$. This figure provides the basis for obtaining the constitutive behaviour from the loading-unloading curve, as it is expressed as a function of parameters that are readily provided by instrumented indentation. However, more information could be obtained from the test if the actual contact area $A$ (or, in other words, the pile-up parameter $c_{p}$ ) were known. The finite element simulations detailed above can provide useful information in this respect, and Fig. 10 shows the dependence of

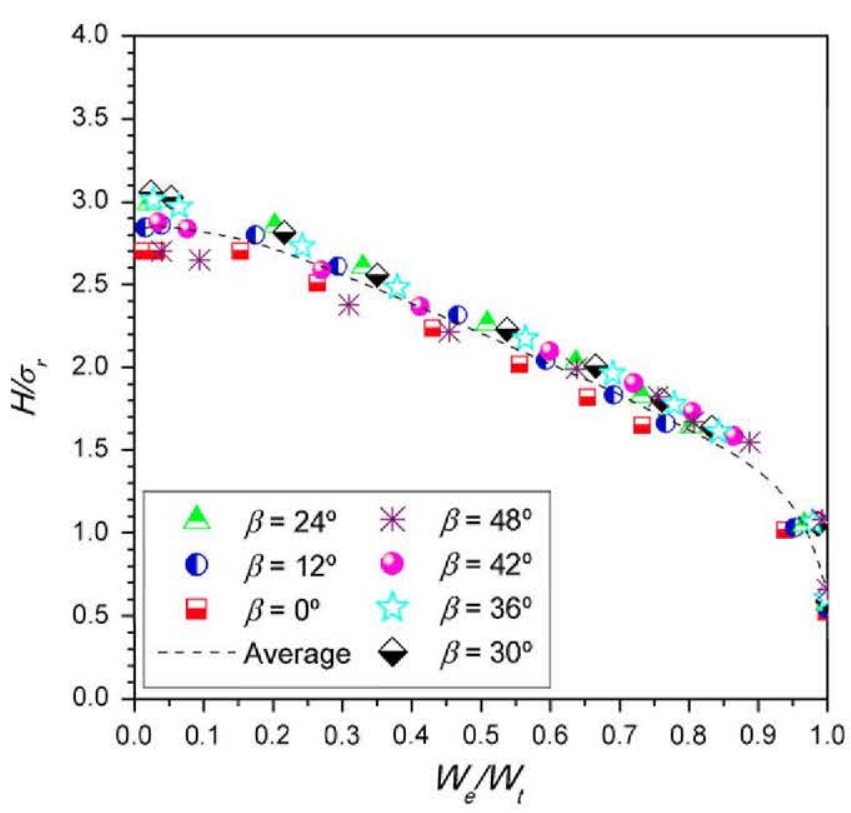

Fig. 8. Evolution of the constraint factor $H / \sigma_{r}$ as a function of the $W_{e} / W_{t}$ ratio for materials with different Drucker-Prager friction angles.

the pile-up parameter on $W_{e} / W_{p}$ obtained from the simulations. For the sake of comparison, the pile-up parameter obtained applying the Oliver and Pharr method [24] to the simulated curves is also included in Fig. 10.

It is obvious that the Oliver and Pharr method leads to very large errors in the actual contact area for very ductile materials $\left(W_{e} / W_{t}<0.5\right)$ and/or materials with low pressure sensitivity, while it can be used to estimate $c_{p}$ if $W_{e} / W_{t}>0.5$.

Based on the above considerations, two main scenarios can be found to determine the mechanical properties of the material from instrumented indentation. If $W_{e} /$ $W_{t}>0.5$, the actual contact area can be obtained from the Oliver and Pharr method. Then $E^{*}$ and $H$ are readily

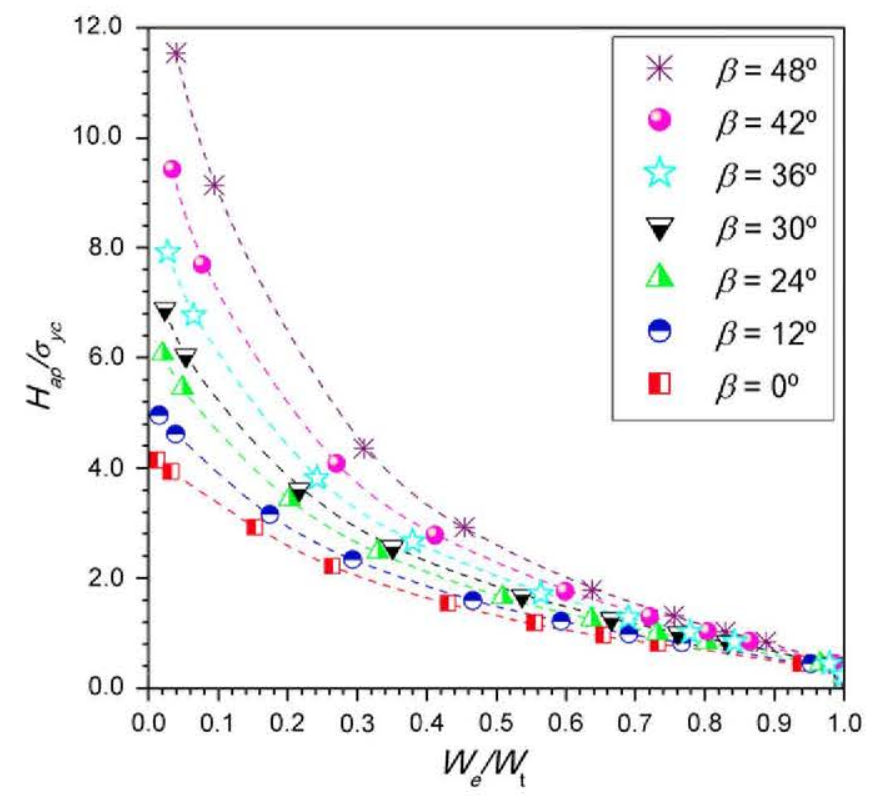

Fig. 9. Evolution of the apparent constraint factor $H_{a p} / \sigma_{y c}$ as a function of $W_{e} / W_{t}$ for different Drucker-Prager friction angles. 


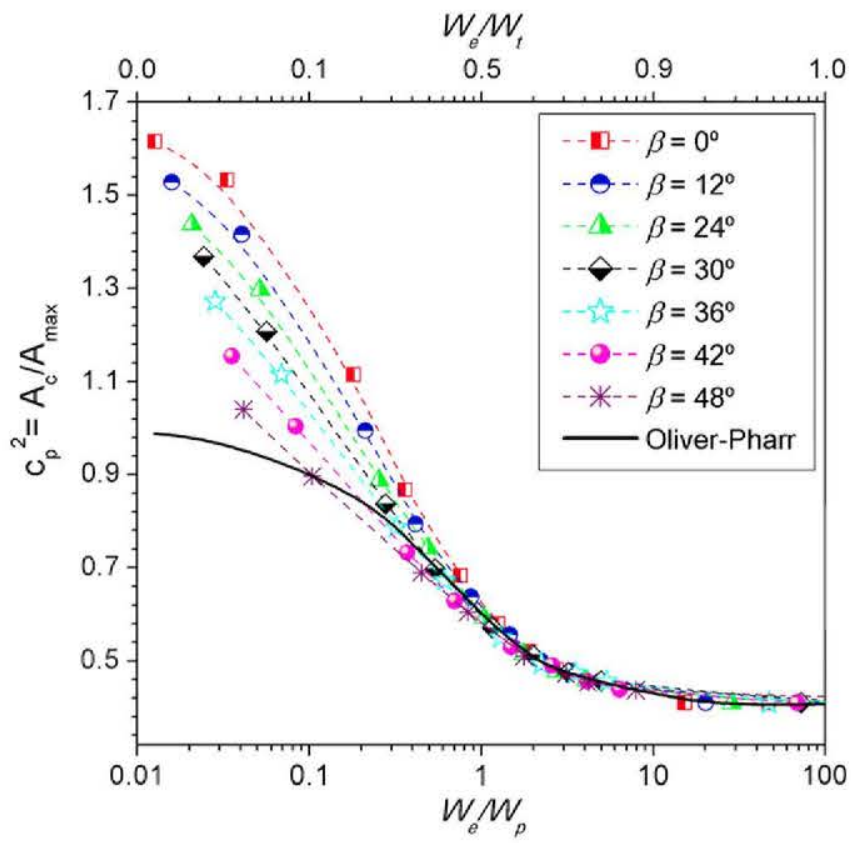

Fig. 10. Evolution of the pile-up parameter as a function of $W_{e} / W_{p}$ and of the friction angle $\beta$. The solid line represents the pile-up estimated by Oliver and Pharr [24].

obtained from Eqs. (6) and (3), respectively. Nevertheless, the pile-up parameter in this regime is insensitive to the friction angle (Fig. 9), and it is not possible to determine both $\sigma_{y c}$ and $\beta$ unless one of them is known in advance. If $\sigma_{y c}$ is known, $\beta$ can be obtained from $H_{a p}$ and $W_{e} / W_{t}$ in Fig. 9. Otherwise, if $\beta$ is known, Fig. 8 provides $\sigma_{r}$ from $W_{e} / W_{t}$, and $\sigma_{y c}$ is obtained from Eq. (14).

If $W_{e} / W_{t}<0.5$, the Oliver and Pharr method does not provide a good estimation of the pile-up parameter, and the best solution is to measure the contact area $A$ after the test by microscopy. Then, $E^{*}$ and $H$ are readily obtained from Eqs. (6) and (3), respectively, while $c_{p}$ is obtained from Eq. (4). $c_{p}$ and $W_{e} / W_{t}$ provide the pressure sensitivity $\beta$ directly from Fig. 9 and, as in the previous case, Fig. 8 provides $\sigma_{r}$ from $W_{e} / W_{t}$, while $\sigma_{y c}$ is obtained from Eq. (14). If the contact area cannot be measured after the test, it is necessary to know a priori at least one of the material properties $\left(E^{*}, \sigma_{y c}\right.$ or $\left.\beta\right)$ to determine the others.

It should be noted that this methodology to determine the elasto-plastic properties of cohesive-frictional materials was based on a single indenter geometry. An alternative approach is the use of two indenters with different geometry, as suggested for pressure-insensitive materials [3,38]. This approach was proposed by Ganneau et al. [27] for cohesive-frictional materials, using two pyramidal indenters with different tip angles (Berkovich and cube corner). Their analysis was only valid, however, for indentations in the fully plastic regime, a category hardly fulfilled by most amorphous materials, as shown experimentally below. In addition, the coefficient of friction between the indenter and the material plays an important role on the constraint factor when the semi-apex angle of the indenter falls below $60^{\circ}[10]$, introducing yet another unknown
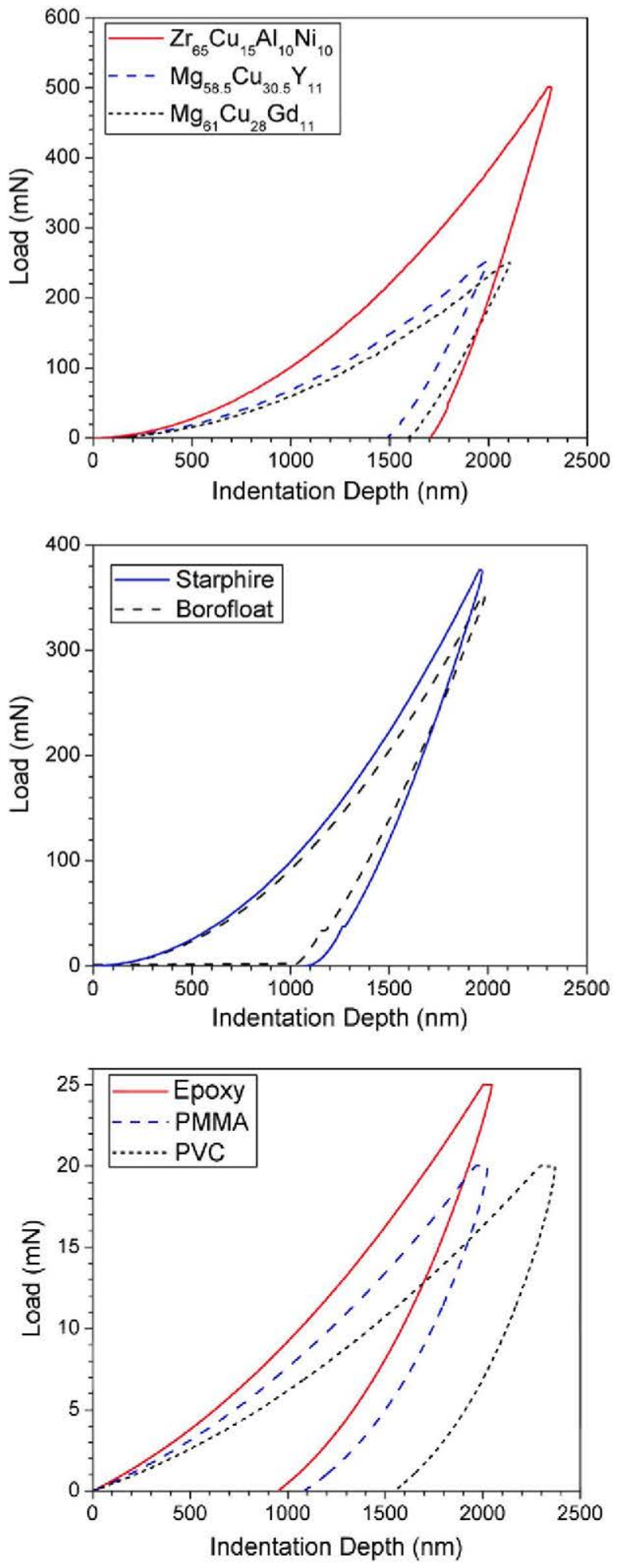

Fig. 11. Representative nanoindentation curves obtained on (a) metallic glasses, (b) ceramic glasses and (c) polymers, respectively.

parameter in the analysis. As an alternative, Seltzer et al. [39] proposed the use of Berkovich and spherical indenters to determine the Drucker-Prager parameters of pressuresensitive elastic perfectly plastic polymers. Nevertheless, the method is limited to materials with $W_{e} / W_{t}>0.5$, because the contact area is estimated by the Oliver and 
Pharr method. It is shown below that this condition is rarely met by amorphous materials, with the exception of some ceramic glasses.

\section{Experimental validation}

\subsection{Materials}

The model described above was applied to the characterization of different amorphous materials: three bulk metallic glasses, two ceramic glasses and three polymers of technological interest. The metallic glasses were provided by the University of Sheffield: one $\mathrm{Zr}$-based metallic glass with composition $\mathrm{Zr}_{65} \mathrm{Cu}_{15} \mathrm{Al}_{10} \mathrm{Ni}_{10}$ and two $\mathrm{Mg}$ based glasses $\left(\mathrm{Mg}_{58.5} \mathrm{Cu}_{30.5} \mathrm{Y}_{11}\right.$ and $\left.\mathrm{Mg}_{61} \mathrm{Cu}_{28} \mathrm{Gd}_{11}\right)$. The ceramic glasses studied were a soda-lime glass (commercial denomination Starphire ${ }^{(\mathbb{B})}$ ) manufactured by PPG (Pittsburg, PA) and a borosilicate glass (commercial denomination Borofloat ${ }^{3} 33$ ) manufactured by Schott Glass $[17,18]$. Finally, the polymers studied were an epoxy resin of aeronautical grade and two thermoplastic polymers of technological interest: PMMA and PVC.

\subsection{Nanoindentation tests}

Indentation experiments were conducted using a Nano Indenter XP (MTS, Nanoinstruments, Oak Ridge, USA) instrument. At least ten indentations were performed in each material, employing a Berkovich tip to an approximate depth of $2 \mu \mathrm{m}$ and an equivalent strain rate of $\dot{\varepsilon}=$ $\dot{h} / h=0.005 \mathrm{~s}^{-1}$, where $h$ and $\dot{h}$ are, respectively, the penetration and the penetration rate of the indenter. Representative indentation curves for the metallic glasses, the ceramic glasses and the polymers are shown in Fig. 11. These amorphous materials cover a very wide range of hardness and modulus values to check the applicability of the new method. Ceramic glasses present the highest hardness and elastic recovery, while metallic glasses show the lowest plasticity index. Polymers display the lowest hardness, but the plasticity index may be very high (epoxy), intermediate (PMMA) or very low (PVC).

The residual imprints in the bulk metallic glasses were measured by scanning electron microscopy (SEM) on a Zeiss EVO MA 15 microscope and by atomic force microscopy (AFM) on a Park XE150 instrument. This was not necessary in the case of the ceramic glasses, because $W_{e} / W_{t}>0.5$, and hence the contact area can be estimated using the Oliver and Pharr method. In the case of polymers, their inherent viscoelastic and viscoplastic behaviour led to a significant recovery of the residual imprint after unloading, and the actual contact area could not be measured. The analysis performed in each class of materials is described below.

\subsection{Bulk metallic glasses}

Metallic glasses showed $W_{e} / W_{t} \approx 0.31$ (Table 1). Since no prior knowledge of their properties was available, the residual imprints were directly measured by SEM and AFM. It is important to notice that the accuracy of this method to determine the mechanical properties depends strongly on the precise determination of the contact area $A$. In this sense, AFM is better suited than SEM, as it provides a topographic image of the residual imprint, from

Table 1

Elasto-plastic material properties obtained from the nanoindentation tests in bulk metallic glasses; literature data for the compressive strength and friction angle are also included for comparison.

\begin{tabular}{|c|c|c|c|c|c|c|c|c|}
\hline \multirow[t]{2}{*}{ Material } & \multicolumn{3}{|c|}{ Indentation data } & \multicolumn{3}{|c|}{ Material properties } & \multicolumn{2}{|l|}{ Literature data } \\
\hline & $H_{a p}(\mathrm{MPa})$ & $W_{e} / W_{t}$ & $c_{p}^{2}$ & $E^{*}(\mathrm{GPa})$ & $\sigma_{y c}(\mathrm{MPa})$ & $\beta\left({ }^{\circ}\right)$ & $\sigma_{y c}(\mathrm{MPa})$ & $\beta\left({ }^{\circ}\right)$ \\
\hline $\mathrm{Zr}_{65} \mathrm{Cu}_{15} \mathrm{Al}_{10} \mathrm{Ni}_{10}$ & $4038+18$ & $0.32+0.01$ & $0.77 \pm 0.01$ & $52+1$ & $1800+100$ & $14 \pm 6$ & $1600-1800[40,41]$ & $<20$ \\
\hline $\mathrm{Mg}_{58.5} \mathrm{Cu}_{30.5} \mathrm{Y}_{11}$ & $2564 \pm 17$ & $0.31+0.01$ & $0.74+0.02$ & $35 \pm 1$ & $942_{-149}^{+84}$ & $29 \pm 5$ & $986 \pm 28[42]$ & - \\
\hline $\mathrm{Mg}_{61} \mathrm{Cu}_{28} \mathrm{Gd}_{11}$ & $2463 \pm 10$ & $0.32 \pm 0.01$ & $0.74 \pm 0.03$ & $35 \pm 2$ & $956_{-107}^{+134}$ & $25 \pm 7$ & $1075 \pm 35[43]$ & - \\
\hline
\end{tabular}

(a) Topography (AFM)

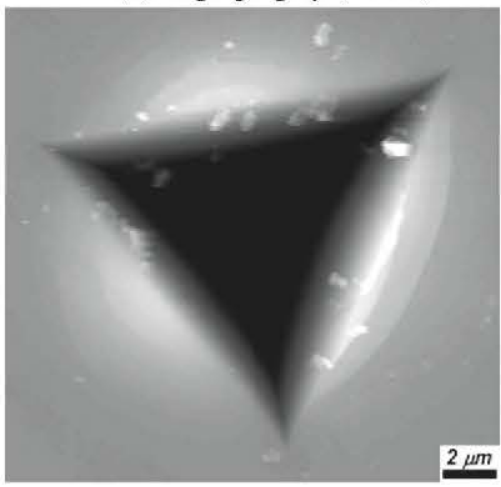

(b) Laplacian (AFM)

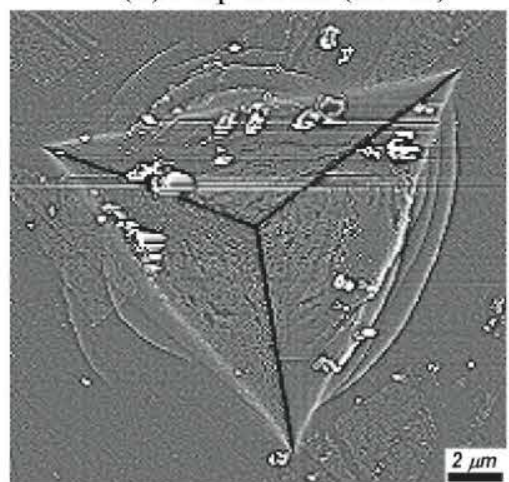

(c) SEM

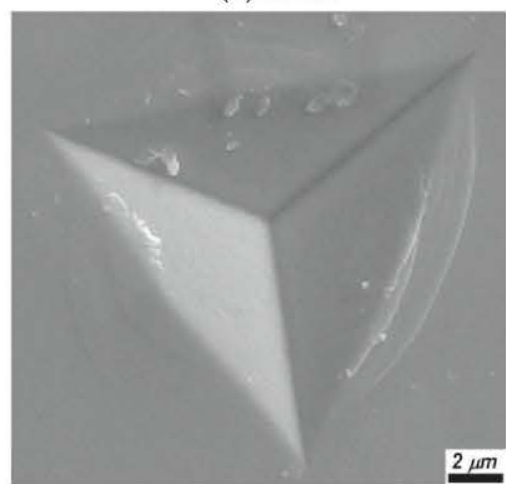

Fig. 12. Images of a residual imprint in the Zr-based metallic glass: (a) topography (AFM); (b) Laplacian (AFM); (c) SEM. 


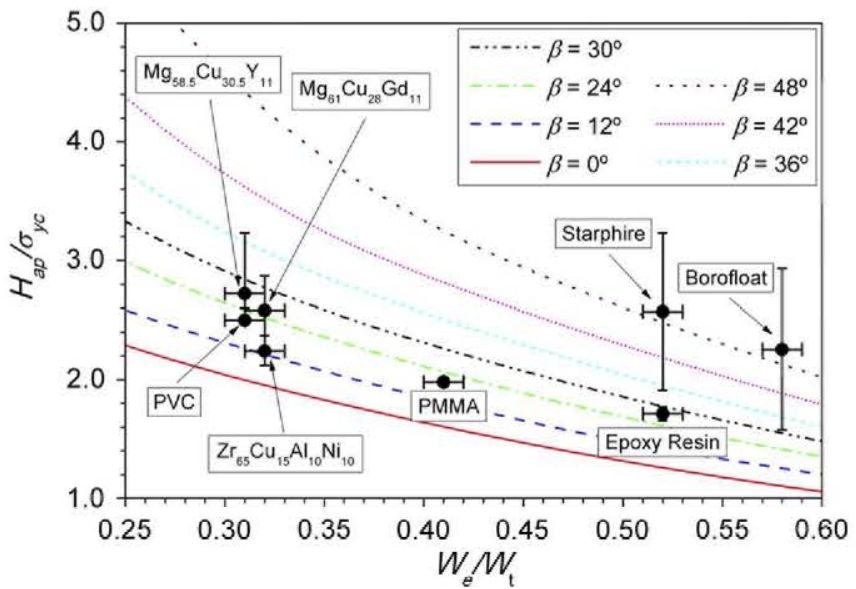

Fig. 13. Experimental values of $H_{a p} / \sigma_{y c}$ as a function of $W_{e} / W_{t}$ for the materials under study, together with the master curves obtained from the numerical simulations.

which the edges of the indentation are more readily obtained through the computation of the Laplacian. Fig. 12 shows three different images of the same residual indentation as measured by AFM (topography and Laplacian) and SEM for the Zr-based glass. The material pile-up, indicative of indentation dominated by plastic deformation, was clearly identified. The images also show the semi-circular shear bands, in the form of overlapping layers of upwardly displaced material, typical of indentations in bulk metallic glasses [16]. The pile-up behaviour makes the sides of the residual imprint bow out, leading to the concavity found in the indentation edges.

Table 1 summarizes the results of the analysis from the values of $H_{a p}, W_{e} / W_{t}$ and $c_{p}$. Bulk metallic glasses lie exactly in the elasto-plastic regime $\left(0.30<W_{e} / W_{t}<0.35\right)$, where the pressure sensitivity has a marked influence on the indentation response. These materials present an important pile-up, even though they are not in the fully plastic regime, so any method based on this assumption (such as that of Ganneau et al. [27] based on slip line theory) and/ or that neglects pile-up effects (such as that of Seltzer et al. [39]) cannot provide accurate results.

The experimental results for $H_{a p} / \sigma_{y c}$ as a function of $W_{e} / W_{t}$ are plotted together with the numerical master curves in Fig. 13. The Zr-based glass is found to have a low pressure dependence with a Drucker-Prager friction angle $\beta<12^{\circ}$. However, the two Mg-based glasses presented moderate pressure sensitivities, with average values of $\beta$ ranging from $24^{\circ}$ to $30^{\circ}$ although the glass containing $\mathrm{Y}$ showed a slightly higher pressure sensitivity. Literature data for the uniaxial compressive strength are also included

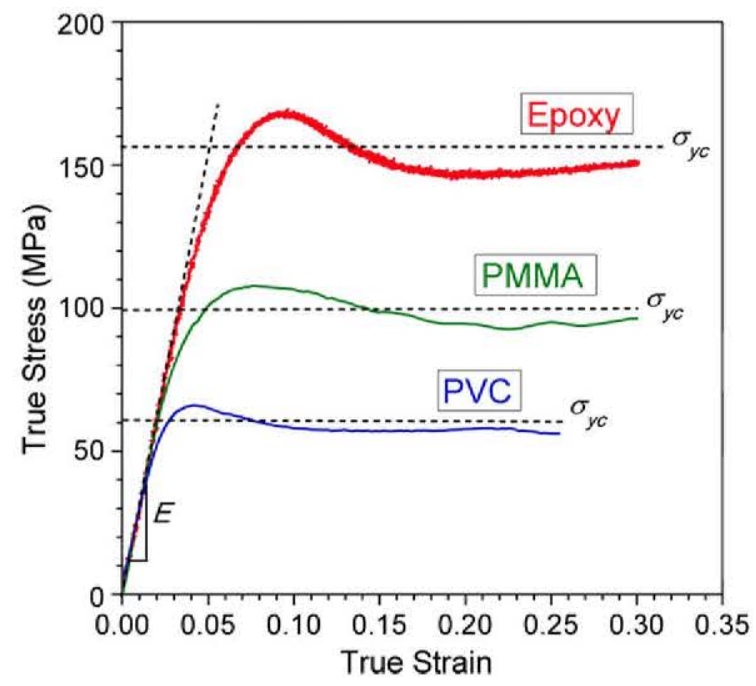

Fig. 14. Compressive stress-strain curves for three polymeric materials: epoxy, PMMA and PVC. The solid lines stand for the experimental results, while the discontinuous lines represent the approximation to an elasto-plastic non-hardening behaviour in each case.

in Table 1 and are in very good agreement with the results obtained from instrumented nanoindentation.

The literature values of friction angle of bulk metallic glasses present a large scatter. They are measured by different methods, including the orientation of the shear bands during uniaxial compression, multiaxial tests in a confining sleeve, or the ratio between tensile and compressive strength. Keryvin et al. [15], Lewandowski and Lowhaphandu [44] and Yuan et al. [45] suggested that $\mathrm{Zr}$-based glass shows limited pressure sensitivity with $\beta<10^{\circ}$. Others authors $[16,46]$ maintain that these materials present a moderate pressure dependence with $\beta \approx 20^{\circ}$. The present results $\left(\beta \approx 14^{\circ}\right)$ and the good agreement in the estimation of the compressive strength support the validity of the method for determining the mechanical properties of metallic glasses from instrumented indentation. In the case of the Mg-based glasses, no reference values could be found in the literature, but the present results suggest a larger pressure sensitivity, with $\beta$ from $24^{\circ}$ to $30^{\circ}$, which is higher for the Y-doped $\mathrm{Mg}$ than for the Gd-doped Mg. Once again, the compressive yield strength derived from indentation was very close to that obtained from conventional uniaxial tests.

\subsection{Ceramic glasses}

The ceramic glasses displayed $W_{e} / W_{t}$ values in excess of 0.5 (Table 2) and were placed in the region where the

Table 2

Elastic modulus and friction angle of ceramic glasses obtained from the nanoindentation tests.

\begin{tabular}{|c|c|c|c|c|c|c|c|}
\hline \multirow[t]{2}{*}{ Material } & \multicolumn{3}{|c|}{ Indentation data } & \multicolumn{2}{|c|}{ Material properties } & \multicolumn{2}{|c|}{ Literature data } \\
\hline & $W_{e} / W_{t}$ & $H_{a p}(\mathrm{MPa})$ & $H^{\mathrm{a}}(\mathrm{MPa})$ & $E^{*}(\mathrm{GPa})$ & $\beta\left({ }^{\circ}\right)$ & $\sigma_{y c}(\mathrm{MPa})$ & $\beta\left(^{\circ}\right)$ \\
\hline Starphire & $0.52 \pm 0.01$ & $4204 \pm 57$ & $7249 \pm 98$ & $61.6 \pm 0.4$ & $45 \pm 7$ & $2000 \pm 600$ & $49[18]$ \\
\hline Borofloat & $0.58 \pm 0.01$ & $4109 \pm 81$ & $7609 \pm 150$ & $77.7 \pm 0.5$ & $45 \pm 5$ & $1750 \pm 450$ & $52[17]$ \\
\hline
\end{tabular}

\footnotetext{
${ }^{\mathrm{a}}$ Using Oliver and Pharr [24].
} 
Table 3

Elastic modulus and friction angle obtained from indentation tests in the polymers.

\begin{tabular}{|c|c|c|c|c|c|c|}
\hline \multirow[t]{2}{*}{ Material } & \multicolumn{2}{|c|}{ Indentation data } & \multirow{2}{*}{$\begin{array}{l}\text { Compression tests } \\
\sigma_{y c}(\mathrm{MPa})\end{array}$} & \multicolumn{2}{|c|}{ Material properties } & \multirow{2}{*}{$\begin{array}{l}\text { Literature data } \\
\beta\left(^{\circ}\right)\end{array}$} \\
\hline & $W_{e} / W_{t}$ & $H_{a p}(\mathrm{MPa})$ & & $E^{*}(\mathrm{MPa})$ & $\beta\left({ }^{\circ}\right)$ & \\
\hline Epoxy & $0.52 \pm 0.01$ & $262+3$ & $153+5$ & $3490 \pm 47$ & $29+2$ & $30[20]$ \\
\hline PMMA & $0.41 \pm 0.01$ & $200 \pm 1$ & $101 \pm 2$ & $3090 \pm 141$ & $20 \pm 2$ & $17[20]$ \\
\hline PVC & $0.31 \pm 0.01$ & $152 \pm 1$ & $61 \pm 0$ & $2950 \pm 173$ & $20 \pm 2$ & $13[47]$ \\
\hline
\end{tabular}

Oliver and Pharr method provides an accurate estimation of the contact area $A$. As explained above, $\beta$ can only be determined under such conditions if the compressive yield strength $\sigma_{y c}$ is known a priori. From the $\sigma_{y c}$ values determined by Chocron et al. [17] and Dannemann [18], it was found that both Starphire and Borofloat glasses present very high pressure sensitivity, $\beta>48^{\circ}$ (Fig. 13). This is in agreement with previous results obtained from multiaxial compression tests, which reported friction angles of $\sim 50^{\circ}$ $[17,18]$.

\subsection{Polymers}

The polymers studied showed $W_{e} / W_{t}<0.5$. Instrumented indentation can only be used to measure the pressure sensitivity if the compressive yield strength is known in advance, since the residual imprint is not representative of the indentation contact area owing to viscoelastic and viscoplastic effects. For this reason, uniaxial compression tests were carried out to measure $\sigma_{y c}$, while nanoindentation provided the pressure sensitivity.

Uniaxial compression tests were carried out at the same strain rate of $0.005 \mathrm{~s}^{-1}$ as the nanoindentation tests. The corresponding stress-strain curves are plotted in Fig. 14, showing typical behaviour for polymers with slight softening after the peak strength. The yield stress is difficult to determine, as it might differ by as much as $10 \%$ from the maximum stress [20], and it was defined as the average between the maximum and the plateau stress in compression (Fig. 14).

Table 3 summarizes the $H_{a p}$ and $W_{e} / W_{t}$ values obtained from the nanoindentation tests, together with the elastic modulus and the friction angle obtained from these data. Both PMMA and PVC showed similar pressure sensitivity, with $\beta \approx 20^{\circ}$, while the epoxy resin presented a slightly higher influence of pressure on the yield stress, with $\beta \approx 29^{\circ}$. This latter value agrees well with those reported by Quinson et al. [20], who measured the yield point of various epoxy resins subjected to different triaxiality levels (uniaxial tension and compression, simple shear and plane strain compression), suggesting a friction angle of $\sim 30^{\circ}$. In the case of PMMA and PVC, other authors [47-49,50] also reported values very close to those obtained in this work.

\section{Conclusions}

A novel methodology based on instrumented indentation was developed to determine the parameters (elastic modulus, compressive yield strength and friction angle) which control the elasto-plastic deformation of amorphous materials with a cohesive-frictional behaviour. The approach is based on the concept of a universal hardness equation for cohesive-frictional materials which results from the assumption of a characteristic indentation pressure which is proportional to the hardness.

The actual universal hardness equation was obtained from a detailed finite element analysis of the process of sharp indentation for a very wide range of material properties. Based on this universal hardness equation, the inverse problem (i.e. how to extract the material parameters $E^{*}, \sigma_{y c}$ and $\beta$ from instrumented indentation) was solved, and the applicability and limitations of the new method analysed. It was found that, if the deformation under the indenter is mainly elastic $\left(W_{e} / W_{t}>0.5\right)$, the pressure sensitivity can only be determined if the yield compressive strength is known in advance. If plasticity controls deformation under the indenter, all the parameters can be extracted from a single pyramidal indentation test, provided the contact area is measured independently (e.g. by AFM). If the actual contact area is not measured independently, the constitutive behaviour can only be determined if one of the three parameters $\left(E^{*}, \sigma_{y c}, \beta\right)$ is known in advance. The method was validated experimentally in a number of amorphous materials (metallic and ceramic glasses as well as polymers) which exhibited cohesive-frictional behaviour and covered a wide range of elastic and plastic properties.

\section{Acknowledgements}

The authors acknowledge the financial support of the Spanish Ministry of Science and Innovation (MAT200914396), the Comunidad de Madrid through the ESTRUMAT programme (S2009/MAT-1585) and European Commission through the Seventh Framework Programme FP7/2007-2013 under Grant Agreement 213371 (MAAXIMUS, www.maaximus.eu). The authors are also indebted to Mr. Plummer and Prof. Todd from Sheffield University for providing the $\mathrm{BMG}$ and to Dr. Chocron from Southwest Research Institute, Texas, for providing the ceramic glasses. MS acknowledges the support from the Comunidad de Madrid for the fellowship to carry out his $\mathrm{PhD}$ thesis.

\section{References}

[1] Dao M, Chollacoop N, Van Vliet KJ, Venkatesh TA, Suresh S. Acta Mater 2001;49:3899.

[2] Cheng YT, Cheng CM. Mater Sci Eng 2004;R44:91. 
[3] Alkorta J, Martínez-Esnaola JM, Gil Sevillano J. J Mater Res 2005;20:432.

[4] Rodney D, Tanguy A, Vadembroucq D. Model Simul Mater Sci Eng 2011;19.

[5] Cheng YT, Cheng CM. Philos Mag Lett 1998;78:115.

[6] Giannakopoulos AE, Larsson PL. Mech Mater 1997;25:1.

[7] Vaidyanathan R, Dao M, Ravichandran G, Suresh S. Acta Mater 2001;49:3781

[8] Narasimhan R. Mech Mater 2004;36:633.

[9] Patnaik MNM, Narasimhan R, Ramamurty U. Acta Mater 2004;52:3335.

[10] Johnson KL. J Mech Phys Solids 1970;18:115.

[11] Johnson KL. Contact mechanics. Cambridge: Cambridge University Press; 1985

[12] Lee YH, Kim JK, Park JS, NAhm SH, Kwon D. J Mater Process Technol 2007;187:794.

[13] Flores KM. Scripta Mater 2001;54:327.

[14] Lund AC, Schuh CA. Acta Mater 2005;51:5399.

[15] Keryvin V, Crosnier R, Laniel R, Hoang VH, Sangleboeuf JC. J Phys D: Appl Phys 2008;41.

[16] Ramamurty U, Jana S, Kawamura Y, Chattopadhyay K. Acta Mater 2005;53:705.

[17] Chocron S, Anderson Jr EA, Nicholls AE, Dannemann KA. J Am Ceram Soc 2010;93:3390.

[18] Dannemann KA, Anderson CE, Chocron S, Spencer JF. J Am Ceram Soc 2012;95:721.

[19] Kinloch AJ, Young RJ. Fracture behaviour of polymers. London: Elsevier Applied Science; 1983.

[20] Quinson R, Pérez J, Rink M, Pavan A. J Mater Sci 1997;32:1371.

[21] Fiedler B, Hojo M, Ochiai S, Schulte K, Ando M. Compos Sci Technol 2001;61:1615.

[22] González C, Llorca J. Compos Sci Technol 2007;67:2795.
[23] Drucker DC, Prager W. Quart Appl Math 1952;10:157.

[24] Oliver WC, Pharr GM. J Mater Res 1992;7:1564.

[25] Tang C, Li Y, Zeng K. Mater Sci Eng A 2004;384:215.

[26] Lockett FJ. J Mech Phys Solids 1963;11:345.

[27] Ganneau FP, Constantinides G, Ulm FJ. Int J Solids Struct 2006:43:1727.

[28] Cheng YT, Cheng CM. Appl Phys Lett 1998;73:614.

[29] Mata M, Anglada M, Alcala J. Philos Mag 2002;49:3781.

[30] Tabor D. Hardness of metals. Oxford: Clarendom Press; 1951.

[31] Li M, Chen WM, Liang NG, Wang LD. J Mater Res 2004;19:73.

[32] Swaddiwudhipong S, Hua J, Tho KK, Liu ZS. Model Simul Mater Sci Eng 2006;14:71.

[33] Wang T, Fang T, Lin Y. Mater Sci Eng A 2007;447:244.

[34] Abaqus Theory Manual. Version 6.10, Simulia; 2011.

[35] Hill R. Theory of plasticity. Oxford: Oxford University Press; 1950.

[36] Marsh DM. J Proc Roy Soc Lond 1964;279:420

[37] Sneddon IN. Int J Eng Sci 1963;3:47.

[38] Luo J, Lin J. Int J Solids Struct 2007;44:5803.

[39] Seltzer R, Cisilino A, Frontini P, Mai Y. Int J Mech Sci 2011;53:471.

[40] Plummer JD, Figueroa IA, Hand RJ, Davies HA, Todd I. J NonCryst Solids 2009;355:335.

[41] Heilmaier M. J Mater Process Technol 2001:117:374.

[42] Zheng Q, Ma H, Ma E, Xu J. Scripta Mater 2006;55:541.

[43] Zheng Q, Cheng S, Strader J, Ma E, Xu J. Scripta Mater 2007;56:161.

[44] Lewandowski J, Lowhaphandu P. Philos Mag A 2002;82:3427.

[45] Yuan F, Prakash V, Lewandowski JJ. Mech Mater 2010;42:248.

[46] Lu J, Ravichandran G. J Mater Res 2003;18:2039.

[47] Yuan J, Hiltner A, Baer E. J Mater Sci 1983;18:3063.

[48] Rabinowitz S, Ward I, Parry J. J Mater Sci 1970;5:29.

[49] Bowden PB, Jukes JA. J Mater Sci 1972;7:52.

[50] Bauwens-Crowet C. J Mater Sci 1973;8:968. 